\title{
Diagnosing Dysfunctional Coping in HIV-Infected Persons
}

\author{
0. H. Ezeh \\ Department of Community Medicine, Ahmadu Bello University, Zaria, Nigeria \\ Email: drohezeh@gmail.com
}

How to cite this paper: Ezeh, O.H. (2019) Diagnosing Dysfunctional Coping in HIV-Infected Persons. Open Journal of Medical Psychology, 8, 36-43. https://doi.org/10.4236/ojmp.2019.82003

Received: March 20, 2019

Accepted: April 27, 2019

Published: April 30, 2019

Copyright (C) 2019 by author(s) and Scientific Research Publishing Inc. This work is licensed under the Creative Commons Attribution International License (CC BY 4.0).

http://creativecommons.org/licenses/by/4.0/
Abstract highly active anti-retro-viral therapy (HAART) to achieve viral suppression. Despite its negative effects on health outcomes, dysfunctional coping remains undiagnosed and untreated in HIV patients. Therefore, it would seem important to diagnose dysfunctional coping in HIV-patients in order to enable health care providers' plan and execute intervention and treatment strategies in a population of HIV-patients/HIV-infected persons, to reduce health problems that may be associated with it. Aims/Objectives: 1) To identify elements of dysfunctional coping in HIV-patients. 2) To screen HIV-infective persons for dysfunctional coping. 3) To assess prevalence of dysfunctional coping among HIV-patients. 4) To assess association of duration of HIV-infection (survivability) with dysfunctional coping in HIV-infected persons. Methods: A cross-sectional descriptive study was carried out, to diagnose dysfunctional coping in HIV-infected patients. Subjects were HIV patients receiving HAART at Ahmadu Bello University Teaching Hospital (ABUTH), Zaria, Nigeria. Over 110 HIV-infected persons were screened for dysfunctional coping, using 28-item Brief Cope Questionnaire. Results: After various forms of statistical analyses were performed on the data, results indicate differing prevalent rates for the four key dimensions of dysfunctional coping derived from the Brief-cope questionnaire, used to measure dysfunctional coping in this study; Denial Coping, 68 (61.8 percent), Behavioral Disengagement Coping, 46 (41.8 percent), Substance Use Coping, 24 (21.8 percent), Self-Blame Coping, 61 (55.5 percent); and composite (Integrated/Combined) dysfunctional coping, 49 (44.5 percent). A total prevalence rate of 44.5 percent was reported. Altogether more than 60 percent of the HIV patients screened positive for one dimension of dysfunctional coping or the other. The qui-square analyses revealed an association of denial coping with duration of HIV-infection/survivability. Conclusion/Recommendation: Overall, the study was able to demonstrate that dysfunctional coping was 
quite common among HIV patients. Dysfunctional coping may be a determinant of health in HIV patients and significantly affect health outcome. It was therefore recommended that interventions focused on coping strategies be integrated into management of HIV patients in order to improve their psychological and physical wellbeing.

\section{Keywords}

HIV Patients, HAART, Diagnosing, Dysfunctional Coping

\section{Introduction}

According to World Health Organization [1], more than 70 million people have been infected with HIV virus and about 35 million people have died of HIV, globally. More than 36.9 million (31.1 - 43.9 million) people were living with HIV at the end of 2017. Also more than 9.4 million people do not know they are living with HIV. Research indicates that personality is a determinant of how an individual copes with stress [2]; for example type-A individuals cope badly under stress. Identifying the correlates of dysfunctional coping in a population of HIV-infected persons may assist in planning appropriate interventions that may help to promote psychological health and improve quality of life.

Coping strategies are psychological patterns that individuals use to manage thoughts, feelings, and actions encountered during various stages of ill health and treatments [3]. Research [4], indicates that coping strategies may be categorized into harmful and helpful varieties. Functional coping strategies may improve physical, mental, emotional and social functioning. It may also increase CD4 counts in HIV patients. Dysfunctional coping strategies are those coping styles that employ avoidance, denial, self-blame, or detrimental ways of coping such as alcohol or drug abuse. Examples of dysfunctional coping include; anxious avoidance, suppression, substance use, medication (escape), etc. Dysfunctional coping may just reduce the symptoms and at the same time maintain and strengthen the health disorders. They are more effective in the short than long term coping process. Dysfunctional coping may also interfere with the individual's ability to unlearn, or break apart, the paired association between the situation and the associated symptoms. They are negative, and serve to maintain or worsen the health disorders. Dysfunctional coping may also impact negatively; the effects of HAART to achieve viral suppression in HIV-infected patients, increase the speed of HIV-disease progression, and may also be counter-productive or have unintended negative health consequences in general. Despite all these adverse effects on health outcomes in the patients, dysfunctional coping remains undiagnosed and untreated. The study therefore, seeks to assess coping skills in HIV patients in order to diagnose dysfunctional coping, prescribe intervention to treat/address and prevent it.

The investigation focuses on four key questions: 
1) What percentage of HIV-infected persons are using dysfunctional coping?

2) How can HIV-infected persons, using dysfunctional coping be identified?

3) How many of them belong to HIV-support group?

4) Is dysfunctional coping related to the duration of HIV-infection/survival?

The main aim of the study was to examine the above questions in HIV-infected persons, since bodily changes associated with HIV-infection may generate severe coping demands.

\section{Aims/Objectives}

1) To identify elements of dysfunctional coping in HIV-patients.

2) To screen HIV-patients for dysfunctional coping.

3) To assess prevalence of dysfunctional coping in HIV-patients.

4) To assess relationship between duration of HIV-infection/survivability and dysfunctional coping in HIV-patients.

\section{Methods}

A cross-sectional descriptive study was conducted. Subjects were 110 (35 males and 75 females) HIV-patients receiving, highly active antiretroviral therapy (HAART) from Ahmadu Bello University teaching hospital, Zaria, Nigeria, who were selected for the study, through convenience/haphazard sampling technique. The 110 patients used in the study, were those who were readily available and were willing to participate actively in the study. The mean age of the patients was 39.8 years, standard deviation 11.6, minimum age 18 years, and maximum 75 years with most, ( $42 ; 38.18$ percent) of the subjects within the age bracket of (36 45) years. Duration of HIV-infection was categorized into; 1 year or less, 2 - 4 years, 5 - 10 years and above 10 years. From literature review, four subscales of Brief-cope questionnaire were employed to assess dysfunctional coping. The four dimensions/aspects of dysfunctional coping were assessed using subscales of Denial, Behavioral disengagement, Substance use, and Self-blame of the Brief-cope questionnaire [5]. In addition, the scores on these subscales were pooled together to generate a broad or composite (Total) dysfunctional coping score for each respondent. Research [6], indicates that Brief-Cope is a highly reliable measure with good validity, demonstrated across samples. It may be used as a screening tool, to determine the best coping-skills intervention for HIV-patients. The Brief-Cope questionnaire is made up of 14 scales each of which assesses the extent to which a subject utilizes a specific coping strategy. The 14 scales include; Active Coping, Planning, Positive Reframing, Acceptance, Humor, Religion, Using Emotional Support, Using Instrumental Support, Self-Distraction, Denial, Venting, Substance Use, Behavioral Disengagement, and Self-Blame. The patients were instructed to rate items on a 4-point Likert-scale, ranging from; 1 "I haven't been doing this at all" to 4 "I've been doing this a lot". Each of the 14 scales had 2 items, total score on each scale ranged from 2 (minimum) to 8 (maximum). Higher scores indicate increased use of that specific coping strategy. Total scores 
on each of the scales were calculated by summing the appropriate items for each scale. Although the whole Brief-Cope was administered, only 4 scales (Denial, Behavioral Disengagement, Substance Use and Self-Blame) were used, for the assessment of dysfunctional coping. The total score of the dimensions of dysfunctional coping was calculated for each respondent.

There was also questionnaire to assess socio-demographics of the subjects including; age, sex, marital status, duration of HIV-infection/survival, HIV-support group patients belonged to, etc. Although the whole Brief-COPE was administered, only the subscales that bordered on dysfunctional coping were scored and used for analyses. The study was approved by ethical committee of the hospital, and consent was gotten from the subjects, all of whom consented to the study procedures.

Various forms of statistical analyses were performed on the data collected, using statistical package for social sciences (SPSS) version 21.

\section{Results}

The study identified (Table 1 ) four ( 1 - 4 above) dimensions of dysfunctional coping, and one composite/combined (5 above; total of the dimensions) dysfunctional coping. Statistical analyses were performed using the four dysfunctional subscales separately, and compositely, to identify their individual and composite/total contributions to coping dysfunction.

The two main demographic groups (Table 2) based on duration of HIV-infection/survivability with the highest frequencies were; 2 - 4 years (38 HIV-patients) and 5 - 4 years (38 HIV-patients).

Table 1. Identification of dimensions of dysfunctional coping from the brief-COPE questionnaire.

\begin{tabular}{ccc}
\hline S/N & Dysfunctional Coping Dimension & Abbreviation \\
\hline 1$)$ & Denial Coping & DN \\
$2)$ & Behavioral Disengagement Coping & BD \\
$3)$ & Substance Use Coping & SU \\
$4)$ & Self-Blame Coping & SB \\
$5)$ & Composite/Combined/Total Dysfunctional Coping & TOT \\
\hline
\end{tabular}

Table 2. Distribution of respondents by duration of HIV-infection.

\begin{tabular}{cccc}
\hline Duration (Years) & Frequency & Percent $(\%)$ & Cum. \\
\hline$<1$ year & 11 & 10.00 & 10.00 \\
$2-4$ years & 38 & 34.55 & 44.55 \\
5 - 10 years & 38 & 34.55 & 79.09 \\
$>10$ years & 23 & 20.91 & 100.00 \\
Total & 110 & 100.00 & \\
\hline
\end{tabular}

Survey Data, 2018. 
Table 3 indicates that the mean scores of HIV patients on dysfunctional coping dimensions were; Denial coping 5.745, Behavioral Disengagement coping 3.254, Substance Use coping 2.536, Self-Blame coping 3.627, and Total/Combined dysfunctional coping 15.163.

Data suggest (Table 4) that prevalence rates of dysfunctional coping dimensions (DN, 61.8 percent; BD, 41.8 percent SU 21.8 percent; SB, 55.5 percent), and that of TOT (Composite), 44.5 percent were generally high.

The demographic group with the highest prevalence of Denial coping was patients with HIV-duration of 2-4 years (Table 5).

Data analyses suggest (Table 6), HIV patients with shorter duration of HIVinfection/survivability were more likely to use Denial (DN) Coping, $\mathrm{P}=0.001$. Data also suggest that as duration of HIV-infection/survivability increases, denial coping decreases.

Table 3. Summarizing dimensions of dysfunctional coping and their composite (total).

\begin{tabular}{cccccc}
\hline Variable & Obs. & Mean & Std. Deviation & Min. & Max. \\
\hline DN & 110 & 5.745455 & 1.893671 & 2 & 8 \\
BD & 110 & 3.254545 & 1.523133 & 2 & 8 \\
SU & 110 & 2.536364 & 1.162702 & 1 & 8 \\
SB & 110 & 3.627273 & 1.507529 & 2 & 8 \\
TOT & 110 & 15.16364 & 3.029578 & 8 & 23
\end{tabular}

Survey Data, 2018.

Table 4. Differing prevalence rates of dimensions of dysfunctional coping and their composite (total).

\begin{tabular}{ccccc}
\hline Variable & Male & Female & Total Prevalence Rate & Percent N = 110 \\
\hline DN & 20 & 48 & 68 & 61.8 percent \\
BD & 14 & 31 & 46 & 41.8 percent \\
SU & 13 & 11 & 24 & 21.8 percent \\
SB & 18 & 43 & 61 & 55.5 percent \\
TOT & 17 & 32 & 49 & 44.5 percent \\
\hline
\end{tabular}

Survey Data, 2018.

Table 5. Prevalence of denial coping by duration of HIV-infection/survivability.

\begin{tabular}{cccc}
\hline HIV-DURATION & Freq. & Percent & Cum. \\
\hline 1 year and less & 9 & 13.24 & 13.24 \\
$2-4$ years & 30 & 44.12 & 57.35 \\
5 - 10 years & 22 & 32.35 & 89.71 \\
More than 10 years & 7 & 10.29 & 100.00
\end{tabular}

Survey Data, 2018 
Table 6. Summary of qui-square analyses of duration of hiv infection by dimensions of dysfunctional coping.

\begin{tabular}{cccc}
\hline Dysfunctional Coping & $X^{2}$ & $\mathrm{P}$ & \\
\hline DN & 11.826 & $\mathrm{P}=0.001$ & $* *$ \\
BD & 0.139 & $\mathrm{P}=0.7096$ & $\mathrm{NS}$ \\
SU & 0.021 & $\mathrm{P}=0.8887$ & $\mathrm{NS}$ \\
SB & 0.102 & $\mathrm{P}=0.7495$ & NS \\
TOT & 1.500 & $\mathrm{P}=0.2207$ & NS \\
\hline
\end{tabular}

Survey Data, 2018.

\section{Discussions}

Of the 110 patients studied none of them belonged to any HIV-support group of people experiencing similar challenges, and research consistently indicates the therapeutic effects of these groups as effective and functional coping impact for HIV patients, which would assist the patients in maintaining some levels of quality of life. Research [7] identifies social support as a protective factor for life stress that may be linked with decreased distress in HIV-infected persons. Recent report suggests a positive association between social support and use of HAART. The findings of this study suggest that prevalence rates (Table 4) of dysfunctional coping dimensions (DN, 61.8 percent; BD, 41.8 percent; SU 21.8 percent; SB, 55.5 percent), and that of TOT (Composite), 44.5 percent were generally high. These findings were consistent with those of previous studies [8] [9] [10]. Dysfunctional coping may be associated with faster HIV-progression, morbidity and mortality. Denial coping was found to be particularly high in this study. It may dispose HIV patients to withdraw from their friends, colleagues, family; hiding their HIV-status from others and even from healthcare providers, leading to poor adherence, substance use etc.

The study notes that dysfunctional coping in HIV-infected persons may be viewed as a spectrum with some of the patients scoring above the mean while others score below the mean in the direction of abnormality. Other factors which may have contributed to high prevalence of dysfunctional coping in HIV-infected persons include; non-compliance/non-adherence, illness perception, personality, subjective interpretation of HIV-infection, and reaction to it, personality and attitudinal factors etc. Dysfunctional coping may add to the cumulative stress loads of patients who frequently use it. It may constitute psychosocial risk factor relating to faster HIV-disease progression.

Also denial coping was the dimension of dysfunctional coping found to be most associated with duration of HIV/survivability. HIV patients with shorter HIV-duration/survivability were more likely to use denial coping than those of longer duration. This may be due to the fact that they were still learning to live positively with the HIV infection; they may not have really adjusted to the HIV. Effective coping requires conscious, goal-directed behavior that may determine 
the ability to live fulfilling life with the continued, chronic challenges of HIVgenerated stress [9].

\section{Conclusion}

Overall, the study has been able to demonstrate that dysfunctional coping, both the individual dysfunctional coping dimensions and the combined/composite dysfunctional coping were highly prevalent among HIV patients; and that dysfunctional coping (particularly denial coping), was associated with shorter HIV duration/survivability. Dysfunctional coping may become psychosocial risk factor relating to faster HIV-progression and spread, and may also constitute one of the determinants of health in HIV patients.

\section{Recommendations}

Therefore interventions targeted at ineffective and dysfunctional coping strategies should be considered part of integrated treatment for HIV patients. Brief-COPE may be used as initial screening tool, the results of which may help determine the best coping skills intervention for HIV patients. HIV-infected persons should be counseled to join support groups, which may help them in building resilience necessary to navigate the difficulties associated with living with the infection. Utilizing HIV-support services e.g. psychotherapy, substance abuse counseling may help to reduce levels of dysfunctional coping and increase levels of functional coping. Multidisciplinary HIV-treatment team, which should include clinical psychologists among other health professionals who would develop appropriate coping strategies that would not only reinforce treatment programs but also assist the patient live fulfilled life, is recommended. Dysfunctional coping in HIV patients may remain undiagnosed and untreated despite its adverse effects on health outcomes. Therefore, all HIV patients assessing care in healthcare out-fit, should be screened for dysfunctional coping.

Assisting HIV patients in building support-network and providing them with special HIV-support at family, neighborhood, community, faith-based, and governmental levels are also highly recommended.

\section{Conflicts of Interest}

The author declares no conflicts of interest regarding the publication of this paper.

\section{References}

[1] World Health Organization (2018) Global Health Observation (GHO) Data. https://www.who.int/hiv/en/ https://doi.org/10.33425/2639-8486.1010

[2] Ezeh, O.H. and Ezeh, C.C. (2018) Prevalence and Determinants of Cardiac Personality Tendencies: An Assessment of Type A Behavioral Patterns among Youths in Kaduna State, Nigeria. Journal of Cardiology and Vascular Research, 2, 1-4.

[3] Science-Direct (2018) Coping Strategies Elsevier B V. 
https://www.sciencedirect.com/

[4] Skinner, E.A. and Edge, K., Altman, J. and Sherwood, H. (2003) Searching for the Structure of Coping: A Review and Critique of Category Systems for Classifing Ways of Coping. Psychological Bulletin, 129, 216-269.

https://doi.org/10.1037/0033-2909.129.2.216

[5] Carver, C.S. (1997) You Want to Measure Coping but Your Protocol Is Too Long: Consider the Brief Cope. International Journal of Behavioral Medicine, 4, 92-100. https://doi.org/10.1207/s15327558ijbm0401_6

[6] Ezeh, O.H. (2016) Prevalence of Premenstrual Syndrome and Coping Strategies among Female Secondary School Students in Zaria. African Journal of Psychological Study of Social Issues (AJPSS), 19, 115-123.

[7] Cohen, S. and Wills, T.A. (1985) Stress, Social Support and the Buffering Hypothesis. Psychological Bulletin, 98, 310-357. https://doi.org/10.1037/0033-2909.98.2.310

[8] Waddell, E.N. and Messeri, P.A. (2006): Social Support, Disclosure and Use of Anti-retroviral Therapy. AIDS and Behavior, 10, 263-272.

https://doi.org/10.1007/s10461-005-9042-x

[9] Balbin, E.G. (2000): Stress and Coping: The Psychoneuroimmunology of HIV/AIDS. Best Practice Research: Clinical Endocrinology and Metabolism, 13, 615-633. https://doi.org/10.1053/beem.1999.0047

[10] Chida, Y. and Vedhara, K. (2009): Adverse Psychosocial Factors Predict Poorer Prognosis in HIV Disease. Brain, Behavior and Immunity, 23, 434-445.

https://doi.org/10.1016/j.bbi.2009.01.013 